\title{
Arbeitsmarkteffekte atypischer Beschäftigung
}

Die Freude über die quantitativen Erfolge auf dem deutschen Arbeitsmarkt ist nicht ungetrübt. Atypische Beschäftigungsverhältnisse wie Leiharbeit, Befristungen und Minijobs haben gegenüber unbefristeten Vollzeitstellen außerhalb der Zeitarbeitsbranche an Bedeutung gewonnen. Über die Bewertung dieser Entwicklung scheiden sich jedoch die Geister. Während einerseits atypische Beschäftigung als wichtige Medizin für den einstmals „kranken Mann Europas" betrachtet wird, steht sie andererseits in der Kritik, reguläre Arbeitsplätze zu verdrängen. Der vorliegende Aufsatz versucht, die Diskussion zu objektivieren und den bisherigen Forschungsstand über wesentliche Arbeitsmarkteffekte der drei Beschäftigungsformen - Leiharbeit, Befristungen und Minijobs - zusammenzutragen.

CHRISTIAN HOHENDANNER, ULRICH WALWEI

\section{Einleitung}

Seit 2005 hat sich die Arbeitsmarktlage spürbar verbessert. Die Zahl der Erwerbstätigen stieg kräftig an und ihr Anteil an der Bevölkerung im erwerbsfähigen Alter legte entsprechend zu. Gleichzeitig nahmen Unterbeschäftigung und Arbeitslosigkeit deutlich ab. Erstmals seit der Wiedervereinigung führte in den Jahren 2008/2009 eine Rezession - und in dem Fall eine besonders schwere - nicht zu einer neuerlichen Rekordarbeitslosigkeit. Der strukturelle Sockel der Arbeitslosigkeit hat sich somit in der jüngeren Vergangenheit verringert. Mit der Verbesserung der Arbeitsmarktlage geht jedoch ein Wandel der Beschäftigungsformen einher. So haben vollzeitnahe, unbefristete Beschäftigungsverhältnisse ohne Zwischenschaltung einer Zeitarbeitsagentur zugunsten sogenannter atypischer Beschäftigungsverhältnisse wie Leiharbeit, Befristungen oder Minijobs relativ an Bedeutung verloren (vgl. z. B. Keller et al. 2011). Mittlerweile gibt es in Deutschland rund 7,4 Mio geringfügige Beschäftigungsverhältnisse (darunter knapp 2,6 Mio. im Nebenjob), 2,7 Mio befristet Beschäftigte sowie 900.000 Leiharbeiter. $^{1}$

Die Ursachen des Wandels sind vielfältig und unterscheiden sich nach den verschiedenen Beschäftigungsformen (Achatz et al. 2012). Dabei beruhen die Veränderungen insgesamt weniger auf einer im Zeitablauf veränderten Zusammensetzung der Erwerbstätigen wie der wachsenden Frauenbeschäftigung oder der zunehmenden Beschäftigung im Dienstleistungssektor (Sachverständigenrat 2009). Vielmehr sind - unabhängig von solchen Struktureinflüssen - Änderungen im Verhalten der Wirtschaftssubjekte als Treiber des Erwerbsformenwandels auszumachen. Dabei wird der Handlungsspielraum der Wirtschaftssubjekte durch arbeitsmarktrelevante Institutionen (wie z. B. dem Arbeitsrecht, den Rahmenbedingungen für die Vereinbarkeit von Familie und Beruf oder auch den Sozialabgaben) beeinflusst. Präferenzen der Beteiligten und Marktmacht in Teilsegmenten bestimmen dann darüber, inwieweit der Handlungsspielraum ausgeschöpft wird.

Die Strukturveränderungen in der Beschäftigungslandschaft werfen insbesondere die Frage auf, welche Arbeitsmarkteffekte damit verbunden sind.

In unserem im Wesentlichen auf Sekundärquellen basierenden Beitrag konzentrieren wir uns auf drei Formen abhängiger Beschäftigung, die im wissenschaftlichen und politischen Diskurs als besonders umstritten gelten: die befristete Beschäftigung, die Leih-/Zeitarbeit und die

1 Die Zahlen beziehen sich auf Juni 2012. Datenquellen Minijobs: Bundesagentur für Arbeit (2013); Leiharbeit: Schmidt/Wüllerich (2013); Befristete Arbeitsverträge: IABBetriebspanel (Berechnungen der Autoren). 
geringfügig entlohnte Beschäftigung (Minijobs). ${ }^{2}$ Außen vor bleibt die sozialversicherungspflichtige Teilzeitbeschäftigung, deren Zuordnung zur atypischen Beschäftigung - gerade auch wegen der vielfach ausgeprägten Arbeitnehmerwünsche - am wenigsten eindeutig ist. In den nachfolgenden Abschnitten stehen individuelle Effekte und Makrowirkungen atypischer Beschäftigung im Vordergrund. Im Einzelnen geht es um Wege aus der Arbeitslosigkeit und in reguläre Beschäftigung, ${ }^{3}$ Veränderungen der Funktionsweise des Arbeitsmarktes und indirekte, über die Lohnentwicklung vermittelte Wirkungen der Beschäftigungsformen auf den Arbeitsmarkt. Im Fazit werden die Ergebnisse zusammengefasst und Forschungslücken aufgezeigt.

\section{Wege aus der Arbeitslosigkeit und Übergänge in reguläre Beschäftigung}

Im Allgemeinen bietet atypische Beschäftigung Arbeitslosen und anderen Nicht-Erwerbstätigen eine niedrige Einstiegsschwelle in ein - wenn auch tendenziell unsicheres und nicht selten auch schlechter bezahltes - Beschäftigungsverhältnis. Aus Sicht der Outsider steht somit die Frage im Vordergrund, inwiefern sie sich über diesen Weg für einen Platz in der Kernbelegschaft empfehlen können. Betrachtet man atypische Beschäftigungsverhältnisse als eine Möglichkeit, längere Zeitspannen der Arbeitslosigkeit zu vermeiden, so können hiermit weitere positive Effekte einhergehen, die mit dem Erhalt der allgemeinen und spezifischen Qualifikationen zusammenhängen. Indem die Entwertung des individuellen Humankapitals vermieden wird, sinkt die Gefahr von Verfestigungstendenzen der Arbeitslosigkeit. Auch aus psychologischer Sicht dürften die Effekte positiv ausfallen, insbesondere wenn man der Erwerbsarbeit neben der reinen Einkommensfunktion weitere Eigenschaften zuspricht wie die soziale oder gesellschaftliche Teilhabe (Gundert/Hohendanner 2011).

Auch Entmutigungseffekte, die vor allem bei dauerhafter Arbeitslosigkeit eine Rolle spielen, treten seltener auf, wenn zusätzliche Beschäftigungsmöglichkeiten existieren. Menschen gewöhnen sich nicht an Unterstützung durch
Dritte, Eigeninitiative und Eigenverantwortung werden gestärkt und der Rückgriff auf staatliche Leistungen oder das soziale Netz muss seltener erfolgen. Damit strahlen die positiven Effekte eines erleichterten Zugangs zu einer Erwerbstätigkeit auch in andere gesellschaftliche Bereiche aus.

Im Folgenden sollen die Übergangseffekte der drei in diesem Beitrag besonders interessierenden atypischen Formen der Beschäftigung diskutiert werden. Dabei werden Übergänge aus Arbeitslosigkeit in Beschäftigung (Brückeneffekte) und Übergänge aus atypischer in „normale“ Beschäftigung (Sprungbretteffekte) unterschieden.

\subsection{Befristungen}

Es gibt nur wenige Untersuchungen, die die Brückenfunktion befristeter Verträge untersuchen, also der Frage nachgehen, inwieweit befristete Verträge dazu beitragen, Arbeitslose in Beschäftigung zu bringen (Hagen 2003a; Gebel 2013): So lässt sich für arbeitslose Personen drei Jahre nach Aufnahme einer befristeten Tätigkeit eine höhere Wahrscheinlichkeit auf eine unbefristete Anstellung ermitteln als bei einer Vergleichsgruppe, die aus der Arbeitslosigkeit direkt nach einer regulären Beschäftigung suchte (Hagen 2003a). Auch Gebel zeigt, dass die Aufnahme eines befristeten Jobs im Vergleich zur kontrafaktischen Situation, arbeitslos zu bleiben und eine unbefristete Stelle zu suchen, die Beschäftigungschancen während der nächsten fünf Jahre erhöht. Zudem erhöht sich in diesem Zeitraum die Wahrscheinlichkeit auf einen unbefristeten Vertrag und einen höheren Lohn (Gebel 2013).

Auch hinsichtlich der Sprungbrettfunktion zeigt sich, dass Befristungen über einen längeren Zeitraum betrachtet $\mathrm{zu}$ Beschäftigungsverhältnissen führen, die langfristig ebenso stabil sind wie unbefristet abgeschlossene (McGinnity et al. 2005; Boockmann/Hagen 2008; Gebel 2010). Befristungen erfüllen häufig die Funktion einer erweiterten Probezeit und können als Brücke in reguläre Beschäftigung fungieren. Dies gilt vor allem für die Gruppe der jüngeren Arbeitnehmer, die hohe Anteile an befristeten Arbeitsverträgen aufweist. Die Neueinsteiger auf dem Arbeitsmarkt verfügen naturgemäß noch nicht über eine große Berufserfahrung, sodass der Probezeitenaspekt von Befristungen im Vordergrund steht.

Die stärkere Nutzung befristeter Arbeitsverträge impliziert aber auch ein höheres individuelles Risiko, aufgrund
2 Eine geringfügig entlohnte Beschäftigung liegt vor, wenn das Arbeitsentgelt aus dieser Beschäftigung regelmäßig im Monat eine Entgeltgrenze von $450 €$ (bis 2012: $400 €$ ) nicht überschreitet. Die wöchentliche Arbeitszeit und die Anzahl der monatlichen Arbeitseinsätze sind dabei unerheblich. Hat eine Person zwei oder mehrere geringfügig entlohnte Beschäftigungsverhältnisse und beträgt das Entgelt hieraus insgesamt mehr als die Entgeltgrenze, so ist keine dieser Beschäftigungen geringfügig. Übt sie neben einer versicherungspflichtigen Beschäftigung eine geringfügige Beschäftigung aus, so werden diese nicht zusammengerechnet mit der Folge, dass diese Zweitbeschäftigung lediglich den pauschalen Abgaben unterliegt.
Jede weitere geringfügige Beschäftigung führt jedoch zur Versicherungspflicht. Oberhalb der Entgeltgrenze gibt es eine Gleitzone (Midijob) zwischen $450 €$ und $850 €$ mit ermäßigten Sozialversicherungsbeiträgen. Die Midijobs werden im Folgenden nicht weiter behandelt.

3 Weitergehende Effekte auf der individuellen Ebene wie z.B. auf Arbeits- und Lebenszufriedenheit, gesellschaftliche Integration oder auch Gesundheit können im Rahmen dieses Beitrags schon allein aus Platzgründen nicht behandelt werden (siehe hierzu z. B. De Cuyper et al. 2008; Gundert/Hohendanner 2011). 
auslaufender Verträge arbeitslos zu werden (Giesecke 2006). Der Gefahr der anschließenden Arbeitslosigkeit steht jedoch die Chance des Übergangs in ein gesichertes Erwerbsverhältnis gegenüber (Boockmann/Hagen 2005). Schließlich besteht die Möglichkeit, dass einem befristeten Arbeitsverhältnis weitere folgen (Giesecke/Groß 2002). Damit deuten sich zwar gewisse Einsperreffekte an - solange es an sicheren Beschäftigungsalternativen für den Einzelnen fehlt, sind solche Befristungsketten aber einem längerfristigen Verbleib in Arbeitslosigkeit vorzuziehen.

Die Beschäftigungsrisiken und -chancen von Befristungen fallen in Abhängigkeit von persönlichen Charakteristika unterschiedlich aus (z. B. Gundert 2007). So ist das anschließende Arbeitslosigkeitsrisiko bei Geringqualifizierten deutlich höher als bei Höherqualifizierten. Dies überrascht allerdings nicht, wenn man sich die allgemeine Arbeitsmarktlage beider Gruppen vor Augen führt. Beide Pole stellen die Qualifikationsgruppen mit den höchsten Anteilen befristeter Beschäftigung (McGinnity et al. 2005; Rudolph 2006). Bei qualifizierten Arbeitnehmern dürfte die Probezeitenfunktion eine größere Rolle spielen, während bei den geringer Qualifizierten eher die Flexibilitätsfunktion befristeter Verträge im Vordergrund steht.

Die mit Befristungen verbundene Lockerung des Kündigungsschutzes könnte dennoch für Personenkreise mit Integrationsproblemen - wie Geringqualifizierte, Personen mit Migrationshintergrund und Menschen mit gesundheitlichen Beeinträchtigungen - eine Möglichkeit zur Arbeitsmarktintegration bieten. Dabei spielen zunächst Kostensenkungspotenziale eine Rolle, die den Einsatz von weniger produktiven Arbeitnehmern für die Unternehmen attraktiver machen. Gebel und Giesecke (2011) zeigen, dass die Lockerung des Befristungsrechts dazu führt, dass vor allem Geringqualifizierte befristet beschäftigt werden. Zugleich finden die Autoren aber nicht, dass sich das Arbeitslosigkeitsrisiko der Geringqualifizierten gegenüber Qualifizierten verringert hat. Die Ergebnisse lassen eher Zweifel an der Brückenfunktion befristeter Beschäftigung gerade für Geringqualifizierte aufkommen.

Finden Übergänge in reguläre Beschäftigung statt, so könnte man zwar von einer erfolgreichen Brückenfunktion sprechen, „Mitnahmeeffekte“ können aber nicht ausgeschlossen werden. Arbeitskräfte mit vergleichsweise geringer Verhandlungsmacht könnten zunächst ohne funktionale Notwendigkeit pauschal befristet werden, obwohl der Arbeitgeber auch unbefristet eingestellt hätte und eine langfristige Anstellung beabsichtigt. Insofern können auch hohe Übernahmequoten nicht uneingeschränkt positiv bewertet werden, weil die kontrafaktische Situation - eine Welt, in der befristete Verträge untersagt sind - nicht beobachtbar ist.

\subsection{Leiharbeit}

Wenngleich deskriptive Befunde zeigen, dass Zeitarbeitsagenturen in nennenswertem Maße aus dem Pool der Ar- beitslosen rekrutieren, ist dies noch kein Beleg dafür, dass sich dadurch die Beschäftigungschancen der Individuen mit Blick auf den allgemeinen Arbeitsmarkt kurzfristig und nachhaltig verbessern (Möller et al. 2012). Um Aussagen dazu treffen zu können, bedarf es eines geeigneten Vergleichsgruppenansatzes von Personen, die aus der Arbeitslosigkeit via Leiharbeit in eine Beschäftigung außerhalb der Zeitarbeitsbranche kamen und solchen, die einen direkten Übergang realisieren konnten. Eine erste Studie dieser Art für Deutschland lieferte keine Evidenz für solche Brückeneffekte der Leiharbeit (Kvasnicka 2008). Allerdings basiert die Untersuchung auf einem kurzen Zeitraum in den 90er Jahren (1994 bis 1996), also einer Periode, in der die Zeitarbeit hierzulande noch eher ein Schattendasein fristete. Angesichts der Expansion der Zeitarbeit in Deutschland haben sich die Verhältnisse aber seitdem deutlich verändert.

Neuere Untersuchungen kommen daher auch zu etwas anderen Ergebnissen. Zwar sind die Sprungbretteffekte als deutlich geringer anzusehen als bei befristeten Arbeitsverträgen. Nach Crimmann et al. (2009) werden lediglich $7 \%$ der Leiharbeiter vom Entleiher übernommen. Lehmer und Ziegler (2010) konnten aber hinsichtlich der Brückenfunktion zeigen, dass ehemals Langzeitarbeitslose, die im zweiten Quartal 2006 in einer Zeitarbeitsagentur tätig waren, gegenüber einer Vergleichsgruppe von im selben Quartal weiterhin arbeitslosen Personen in der Folge eine erhöhte Beschäftigungswahrscheinlichkeit aufweisen. Ein Jahr später liegt die Differenz bei 17 Prozentpunkten. Bestätigt wird die Brückenfunktion der Arbeitnehmerüberlassung dann noch einmal von Lehmer (2012), der mithilfe verschiedener Matching-Verfahren geringe, aber signifikant positive Effekte ermittelt. Da sich diese Untersuchung auf den Zeitraum bis 2008 erstreckt, kann er außerdem zeigen, dass sich die Chancen des Übergangs von Leiharbeit in Branchen außerhalb der Zeitarbeitsbranche in wirtschaftlich ungünstigeren Zeiten verschlechtern.

\subsection{Geringfügig entlohnte Beschäftigung (Minijobs)}

Bei keiner anderen Beschäftigungsform ist der Kontext der Erwerbsbiografie so bedeutend wie bei der geringfügig entlohnten Beschäftigung (Minijobs). Denn für viele Minijobber steht der Übergang in ein Normalarbeitsverhältnis gar nicht im Vordergrund. So zeigen Registerumfragen, dass $42 \%$ der ausschließlich geringfügig entlohnten Beschäftigten 2010 entweder Schüler und Studenten oder Rentner waren (Körner et al. 2013). Dazu kommt bei Hausfrauen/ Hausmännern (ebenfalls $42 \%$ der geringfügig entlohnten Beschäftigung), dass die Rahmenbedingungen (z. B. fehlende Kinderbetreuung) und Erwerbsanreize (z. B. Ehegattensplittung und Steuerfreiheit der Minijobs) einen Übergang in eine sozialversicherungspflichtige Beschäftigung gar nicht nahe legen. Minijobs sind in vielen Fällen von 
vornherein als Zusatzverdienst im Haushaltskontext oder als Nebenbeschäftigung angelegt.

Minijobs zeichnen sich durch eine vergleichsweise hohe Fluktuation aus. Betrachtet man die Gruppe der ausschließlich geringfügig entlohnten Beschäftigten, ist die Fluktuation doppelt so hoch wie bei voll sozialversicherungspflichtig Beschäftigten (Kalina/Voss-Dahm 2005). Dabei liegen zu den Brückeneffekten der Minijobs keine eindeutigen Befunde vor. Einige Untersuchungen zeigen, dass Minijobs Dequalifizierungsprozessen während der Arbeitslosigkeit entgegenwirken sowie die Verbleibschancen am Arbeitsmarkt erhöhen und damit Übergänge in eine sozialversicherungspflichtige Beschäftigung erleichtern können (Caliendo/Wrohlich 2010). Auch die Ergebnisse von Freier und Steiner (2008) deuten zumindest darauf hin, dass Minijobs die Dauer der Arbeitslosigkeit verkürzen könnten. Andere Studien konnten jedoch so gut wie gar keine Brückeneffekte nachweisen. Dingeldey et al. (2012) finden keine Hinweise, dass sich durch die Aufnahme eines Minijobs die Wahrscheinlichkeit erhöht, den ALG-II-Bezug zu verlassen. Ähnliche Ergebnisse finden sich bei Freier und Steiner (2010) für arbeitslose Männer in Westdeutschland.

Nach dem Teilzeit- und Befristungsgesetz gelten für geringfügig Beschäftigte die gleichen arbeitsrechtlichen und kollektivrechtlichen Standards wie für regulär Beschäftigte. Allerdings werden diese nicht immer eingehalten, wie aktuelle Erhebungen zeigen (Bachmann et al. 2012). Nur eine Minderheit nimmt Pausenzeiten, bezahlten Urlaub oder Lohnfortzahlung bei Feiertagen oder Krankheit in Anspruch. Durch die bei Arbeitnehmern wie Arbeitgebern zum Teil verbreitete Meinung, „der Minijob sei eben nur „Mini“ und damit eine abgespeckte Form des normalen Beschäftigungsverhältnisses“ (Voss/Weinkopf 2012, S. 9), können sich letztlich Kosten- und Flexibilitätsvorteile für die Arbeitgeber ergeben. Damit wird deutlich, dass bei Minijobs weniger die Screeningfunktion als vielmehr Flexibilitäts- und Kostenargumente im Vordergrund stehen.

Der Integrationswirkung von Minijobs ist also mit Skepsis zu begegnen, wenn man den Übergang in ein Normalarbeitsverhältnis als oberstes Ziel betrachtet. Andererseits ergeben sich Möglichkeiten zur verstärkten Partizipation sowie zur verbesserten Vereinbarkeit unterschiedlicher Lebensinhalte. Besonders problematisch erscheint die Tatsache, dass durch Minijobs keine Sozialversicherungsansprüche erworben werden. Damit fehlt den Beschäftigten insbesondere bei einem längeren Verbleib in dieser Erwerbsform eine wichtige Säule der finanziellen Absicherung. Aus diesem Grund ist die Wahrscheinlichkeit groß, dass Menschen, die lange in geringfügiger Beschäftigung verharren (müssen), im Alter auf keine eigenständige Alterssicherung werden zurückgreifen können.

\section{Veränderung der Funktionsweise des Arbeitsmarktes}

Auch auf der Makroebene des Arbeitsmarktes können atypische Beschäftigungsverhältnisse vielfältige Effekte haben. Dabei ist insbesondere eine veränderte Funktionsfähigkeit des Arbeitsmarktes ins Blickfeld zu nehmen. Drei Aspekte sind von besonderer Bedeutung (Boockmann/Hagen 2005): die Anpassung an wirtschaftliche Schwankungen, die Verbesserung von Matchingprozessen sowie die Kostenvorteile atypischer Beschäftigung. Letztere können zu einer höheren Aufnahmefähigkeit des Arbeitsmarktes beitragen, bergen aber gleichzeitig auch das Risiko der Verdrängung regulärer Beschäftigung.

\subsection{Anpassung an wirtschaftliche Schwankungen}

Die Möglichkeit, Beschäftigte flexibel an den Arbeitskräftebedarf anzupassen, gilt als eine zentrale Funktion atypischer Beschäftigung. In dynamischen Arbeitsnachfragemodellen lässt sich zeigen, dass Betriebe mittels atypischer Beschäftigung ihre Beschäftigtenzahlen schneller an wirtschaftliche Schwankungen anpassen können (Bentolila/ Saint-Paul 1992; Nunziata/Staffolani 2007). Empirisch lassen sich für Deutschland Hinweise finden, dass befristete Verträge die Anpassungsgeschwindigkeit der Personalkapazität an den Personalbedarf in den Betrieben erhöhen (Hagen 2003b; Pfeifer 2009).

Auch wenn keine vergleichbaren Analysen der Anpassungsgeschwindigkeit von Leiharbeit vorliegen, gilt Leiharbeit als das Flexibilitätsinstrument schlechthin. Leiharbeit kann im Gegensatz zu befristeten Verträgen auch bei unerwarteten Schocks ad hoc angepasst werden, da nicht erst das Ende der Vertragsdauer abgewartet werden muss (Boockmann/Hagen 2001). Demzufolge eignet sie sich auch als ökonomischer Frühindikator, da sich das konjunkturelle Auf und Ab unmittelbar auf die Nachfrage nach Leiharbeitern niederschlägt (Jahn/Bentzen 2012). Die Anpassungsfunktion von Leiharbeit und befristeter Beschäftigung hat sich gerade in der aktuellen Finanz- und Wirtschaftskrise bestätigt (Hohendanner 2010; Schmidt/Wüllerich 2013). Auch Minijobs erhöhen die Flexibilität, indem das Arbeitsvolumen auf viele kleine Beschäftigungsverhältnisse aufgeteilt wird, die je nach Arbeitsanfall eingesetzt werden (Hohendanner/Stegmaier 2012). Flexible Beschäftigungsformen erfüllen insofern eine Schutzfunktion für die Stammbelegschaft, da sie als Puffer für Marktschwankungen fungieren (z. B. Pfeifer 2009; Hohendanner 2012).

\subsection{Matchingprozesse am Arbeitsmarkt}

Vor allem durch befristete Verträge kann die Matchingeffizienz am Arbeitsmarkt verbessert werden (Boockmann/ Hagen 2008): Befristete Verträge erhöhen zwar die Wahrscheinlichkeit deutlich, dass innerhalb eines Zeitraums von 
zwei Jahren eine Beschäftigung endet. In den darauffolgenden Jahren werden befristete Verträge jedoch seltener beendet als Beschäftigungsverhältnisse, die unbefristet begannen. Diese Ergebnisse können darauf hindeuten, dass befristete Verträge vor allem als Screeninginstrument genutzt und Sortierprozesse am Arbeitsmarkt verbessert werden. Bei geringen Kündigungskosten befristeter Verträge können schlechte Matches schneller aufgelöst und Sortierprozesse beschleunigt werden. Dadurch können sich letztlich für beide Seiten - für Arbeitnehmer wie Arbeitgeber - Vorteile ergeben, indem die jeweils passenden Arbeitgeber und $\mathrm{Ar}$ beitnehmer schneller zueinanderfinden.

Während bei befristeten Arbeitsverträgen beide Vertragsparteien selbst aktiv werden müssen, fungiert im Falle der Leiharbeit die Zeitarbeitsagentur als Vermittlungsagent. Gerade bei geringqualifizierten Personen mit Vermittlungshemmnissen kommt dieser spezifischen Form der „privaten Arbeitsvermittlung“ eine zentrale Rolle zu. Die Zeitarbeit hat bei den Veränderungen am Arbeitsmarkt in den letzten Jahren eine wichtige Rolle gespielt (Schmidt/Wüllerich 2013): Sowohl der Beschäftigungsrückgang in der Wirtschaftskrise 2008/2009 als auch der Beschäftigungsaufbau in den Jahren 2010/2011 gingen zu weiten Teilen auf die Arbeitnehmerüberlassung zurück. ${ }^{4}$ Insofern kann Zeitarbeit neben der Möglichkeit des Screenings im Betrieb eine wichtige Matchingfunktion am Arbeitsmarkt erfüllen. Die relativ geringen Brückeneffekte der Leiharbeit legen jedoch nahe, dass Zeitarbeit in erster Linie ein Flexibilitätsinstrument der Betriebe ist, um wirtschaftliche Volatilitäten abzufedern. Auch die Studien zu Brückeneffekten bei Minijobs deuten darauf hin, dass Minijobs aus betrieblicher Sicht eher ein Flexibilitäts- oder Kostensenkungsinstrument darstellen und seltener die Funktion erfüllen, Matchingprozesse am Arbeitsmarkt zu verbessern.

\subsection{Substitutionseffekte}

Eine zentrale arbeitsmarktpolitische Frage ist, ob atypische Arbeitsverhältnisse zusätzlich sind oder ob Arbeitgeber mit deren Einsatz reguläre Arbeitsverhältnisse ersetzen. Diese Frage ist deshalb so entscheidend, weil davon die Einordnung potenzieller Brücken- und Sprungbretteffekte und damit die generelle Einschätzung der arbeitsmarktpolitischen Funktion der Beschäftigungsformen abhängen. Bei der Beantwortung der Frage stößt die empirische Forschung bislang jedoch an Grenzen. Eine rein deskriptive Betrachtung der Entwicklung atypischer und regulärer Beschäftigungsverhältnisse kann zwar Hinweise geben, genügt jedoch nicht als Nachweis für Substitutionsprozesse, da es sich um zufällige gegenläufige oder parallele Entwicklungen handeln kann (Bachmann et al. 2013). Auch die vorliegenden ökonometrischen Analysen liefern bislang keine eindeutigen Befunde. Im Folgenden werden die wenigen verfügbaren deskriptiven und ökonometrischen Analysen zu Leiharbeit, Minijobs und befristeten Verträgen vorgestellt.

\subsubsection{Leiharbeit}

Promberger (2012) untersucht mittels deskriptiver Analysen des IAB-Betriebspanels 1998 bis 2003 potenzielle Substitutionsprozesse zwischen Leiharbeit und Beschäftigung außerhalb der Zeitarbeitsbranche. Der Autor spricht von Substitution „wenn in einem Betrieb die reguläre Beschäftigung sinkt, während die Leiharbeit wächst oder stagniert, oder wenn die reguläre Beschäftigung stärker sinkt als die Leiharbeit." (Promberger 2012, S.196). Entsprechend dieser Definition fanden im betrachteten Zeitraum von 1998 bis 2003 in rund einem Viertel aller Betriebe, die Leiharbeit nutzen, Verdrängungsprozesse statt. Auch Crimmann et al. (2009) sowie Baumgarten und Kvasnicka (2012) betrachten in ähnlicher Weise den Auf- und Abbau von regulären Arbeitskräften und Leiharbeitern in den Betrieben. Die Autoren betonen jedoch, dass mit einer solchen Betrachtung keinesfalls Substitutionseffekte kausal identifiziert werden können. Jahn und Weber (2013) weisen auf das unterschiedliche Verständnis des Substitutionsbegriffes hin. Als problematisch sehen die Autoren die Messung von Substitution vor allem an, wenn atypische Beschäftigungsverhältnisse zur Erprobung neuer Mitarbeiter fungieren. Hat Substitution stattgefunden, wenn ein Leiharbeitsverhältnis anstelle eines regulären Arbeitsverhältnisses zur Erprobung eingesetzt wird? In beiden Fällen entsteht im Anschluss eine reguläre Beschäftigung oder das Arbeitsverhältnis wird in der Probezeit bzw. im Rahmen der Arbeitnehmerüberlassung aufgelöst.

Auch stellt sich die Frage, ob bei hohen Suchkosten oder einem sehr kurzfristigen Personalbedarf tatsächlich jede Einstellung eines Leiharbeiters die Einstellung einer regulären Arbeitskraft verhindert hätte, oder eher als zusätzlich anzusehen wäre, da angesichts der Situation eben kein reguläres Arbeitsverhältnis entstanden wäre. Selbst wenn Substitutionsprozesse eindeutig vorliegen, könnte es sein, dass im Wettbewerb ohne den Rückgriff auf die Zeitarbeit noch mehr reguläre Mitarbeiter ihre Stelle verloren hätten. Jahn und Weber (2013) verwenden den Substitutionsbegriff daher in einem umfassenderen Sinne, indem sie die Wirkung der Expansion des Zeitarbeitssektors gesamtwirtschaftlich im Rahmen eines makroökonometrischen Modells unter-

\footnotetext{
4 Die jüngsten Entwicklungen deuten jedoch auf einen gewissen Sättigungseffekt in der Zeitarbeit hin (Schmidt/ Wüllerich 2013): Während der Anteil der Zeitarbeit am Aufbau sozialversicherungspflichtiger Beschäftigung im Jahr 2010 noch bei $53 \%$ lag, reduzierte sich der Anteil im Jahr 2011 auf 17 \%. Im Juni 2012 verzeichnete die Zeitarbeit sogar einen negativen Beitrag am Wachstum von etwa $5 \%$. Inwieweit der Bedeutungsrückgang der Zeitarbeit beim Beschäftigungsabbau auf neue Regulierungen wie den Branchenmindestlohn zurückzuführen ist, ist erst noch abzuwarten. Auch die neuen Tarifverträge zu Branchenzuschlägen und die vielfach diskutierten Werkverträge als mögliches Ausweichinstrument könnten den Einsatz der Zeitarbeit begrenzen.
} 
suchen. Damit lassen sich neben den dargestellten Mechanismen weitere direkte und indirekte Wirkungsweisen betrachten. So könnte die Expansion des Zeitarbeitssektors auch Auswirkungen auf den privaten Konsum haben, die Investitionsnachfrage der Unternehmen verändern, oder zu Verdrängungsprozessen auf Produktmärkten führen. Jahn und Weber argumentieren, dass gerade aufgrund der komplexen Wirkungsweisen eine gesamtwirtschaftliche Analyse von Substitutionseffekten erforderlich ist. In ihrer Analyse auf Basis eines makroökonometrischen Modells identifizieren die beiden Autoren sowohl einen Substitutionseffekt als auch einen positiven Effekt auf die gesamtwirtschaftliche Beschäftigung. Bei einem Anstieg der Leiharbeiterzahl um 200.000 wären dabei etwa 100.000 Beschäftigungsverhältnisse außerhalb des Zeitarbeitssektors verdrängt, aber zugleich insgesamt 100.000 zusätzliche Beschäftigungsverhältnisse geschaffen worden.

Garz (2012) untersucht hingegen mittels Fixed-Effects Modellen auf Basis von SOEP-Daten 2001-2009 die Beschäftigungs- und Lohneffekte der Reform der Leiharbeit von 2004. Demnach hat die Reform die individuelle Wahrscheinlichkeit, in Leiharbeit beschäftigt zu sein, deutlich erhöht und die Chance auf reguläre Beschäftigung deutlich verringert. In der Summe zeigen sich jedoch keine gesamtwirtschaftlichen Beschäftigungseffekte. Die Ergebnisse deuten darauf hin, dass reguläre Beschäftigung durch Leiharbeit ersetzt wurde, aber keine zusätzlichen Jobs geschaffen wurden. Darüber hinaus zeigt die Studie, dass das Lohndifferenzial - und damit Abweichungen vom Equal Pay-Prinzip - zwischen regulär Beschäftigten und Leiharbeitern über die Zeit zugenommen hat.

\subsubsection{Minijobs}

Die bisherige Literatur zu Substitutionseffekten zwischen sozialversicherungspflichtiger Beschäftigung und Minijobs liefert zwar Hinweise, aber keine eindeutigen kausalen Befunde. Der sprunghafte Anstieg der Minijobs und der Rückgang der sozialversicherungspflichtigen Beschäftigung nach der Reform der geringfügigen Beschäftigung im Jahr 2003 könnten als Indiz für Substitutionsprozesse gewertet werden. Allerdings könnte der Anstieg auch auf eine Umwandlung von Schwarzarbeit in Minijobs zurückzuführen sein (Greve et al. 2007). Eine jüngere Umfrage kommt zu dem Schluss, dass Schwarzarbeit innerhalb von Minijobs ein verbreitetes Phänomen darstellt: „Schwarzarbeit hat unter den Beschäftigten in Minijobs weniger den Charakter eines „Kavalierdelikts", sondern gilt als Ausweis von Engagement, Flexibilität und Vertrautheit mit dem Arbeitgeber - und ist nahezu eine soziale Norm“ (Wippermann 2012, S. 61). Hohendanner und Stegmaier (2012) gehen in ihren Analysen über die deskriptive Betrachtung hinaus und finden auf Basis von Fixed-Effects Regressionen einen negativen $\mathrm{Zu}$ sammenhang zwischen der Anzahl geringfügiger und sozialversicherungspflichtiger Beschäftigung. Dennoch erlaubt die Studie keine kausale Interpretation der Ergebnisse.
Der negative Zusammenhang zwischen beiden Beschäftigungsformen könnte sich auch durch eine innerbetriebliche Zunahme sozialversicherungspflichtiger und einem Rückgang geringfügiger Beschäftigung erklären. Dies könnte der Fall sein, wenn Minijobs in reguläre Beschäftigungsverhältnisse umgewandelt werden. Allerdings zeigen die bisherigen Studien, wie oben beschrieben, dass Übergänge in sozialversicherungspflichtige Beschäftigung eher selten sind. In der Studie von Hohendanner und Stegmaier (2012) zeigt sich, dass kleine Betriebe häufig einen hohen Anteil an Minijobs aufweisen und dass Hinweise auf Substitutionseffekte eher in kleinen Betrieben zu finden sind. Diese Beobachtung deckt sich mit der Studie des RWI (Bachmann et al. 2013), in der gezeigt wird, dass Betriebe mit einem hohen Anteil an Minijobs, Minijobs seltener in reguläre Beschäftigungsverhältnisse umwandeln. ${ }^{5}$

Zwei weitere Studien nähern sich dem Thema, indem sie die sogenannten Eigen- und Kreuzlohnelastizitäten von Minijobs und regulärer Beschäftigung ermitteln (Jacobi/ Schaffner 2008; Freier/Steiner 2010). Eigenlohnelastizitäten geben an, wie stark eine Lohnerhöhung innerhalb der Gruppe der geringfügig oder regulär Beschäftigten zu einem Beschäftigungsabbau führt. Kreuzlohnelastizitäten zeigen, wie stark reguläre Beschäftigung auf- oder abgebaut wird, wenn sich die Löhne der Minijobber ändern. Jacobi und Schaffner (2008) ermitteln, dass sich die Kreuzlohnelastizität, d.h. die Substituierbarkeit zwischen Minijobs und regulärer Beschäftigung, nach der Reform 2003 erhöht hat. Freier und Steiner (2010) hingegen finden eine sehr niedrige Kreuzlohnelastizität, was wiederum auf geringe Substitutionseffekte hindeutet.

\subsubsection{Befristete Beschäftigung}

Studien, die Hinweise auf Substitutionseffekte zwischen befristeter und regulärer Beschäftigung identifizieren, existieren vor allem in Form von international vergleichenden Länderstudien (Kahn 2010; Gebel/Giesecke 2011; Dieckhoff/Steiber 2012). Kahn (2010) untersucht die Auswirkungen von Arbeitsmarktreformen in neun europäischen Ländern (darunter Deutschland) für temporär und permanent Beschäftigte im Zeitraum 1994 bis 2001. Er kommt zu dem Ergebnis, dass die Liberalisierung temporärer Beschäftigung bei simultaner Beibehaltung der Regulierung für dauerhaft Beschäftigte zwar dazu geführt hat, dass temporäre Beschäftigung zugenommen hat, sich aber insgesamt kein Effekt auf die Gesamtbeschäftigung zeigt. Kahn interpretiert das Ergebnis als einen deutlichen Hinweis auf Substitutionsprozesse zwischen unbefristeter und befristeter Beschäftigung. Nunziata und Steffolani (2007) finden in ihrer Analyse von zehn europäischen Ländern (darunter Deutschland) hin-

5 Dieser Zusammenhang zeigt sich im Übrigen auch bei befristeter Beschäftigung (Hohendanner/Gerner 2010). 
gegen heraus, dass die Deregulierung befristeter Verträge auch das Niveau an unbefristeter Beschäftigung erhöht hat und befristete Verträge eine Sprungbrettfunktion erfüllen. Im Gegenzug identifizieren die Autoren allerdings einen Substitutionseffekt zwischen regulärer Beschäftigung und Leiharbeit. Auch die Ergebnisse von Dieckhoff und Steiber (2012) liefern Hinweise auf positive Beschäftigungseffekte der Liberalisierung temporärer Verträge.

\section{Fazit}

Dass atypische Beschäftigungsverhältnisse in den letzten Dekaden an Bedeutung gewonnen haben, ist unbestritten. Über ihre Bewertung sowie die positiven wie negativen Effekte auf Beschäftigung und Arbeitsmarkt besteht jedoch nicht nur in der öffentlichen Diskussion weitgehend Uneinigkeit. Auch in der wissenschaftlichen Literatur finden sich eine Vielzahl teils widersprüchlicher Befunde, aber keine klaren Ergebnisse. Wir haben den Versuch unternommen, das Puzzle zusammenzusetzen, auch wenn das Bild - angesichts der Fülle an Literatur und der dennoch bestehenden Forschungslücken - unvollständig bleiben muss.

Hinsichtlich der Übergänge in reguläre Beschäftigung zeigen die vorliegenden Studien für die verschiedenen Beschäftigungsformen ein differenziertes Bild. Befristete Arbeitsverträge erfüllen häufig die Funktion einer erweiterten Probezeit und fungieren nicht selten als Brücke in reguläre Beschäftigung. Allerdings geht mit der stärkeren Nutzung befristeter Arbeitsverträge auch ein höheres individuelles Risiko einher, aufgrund eines auslaufenden Vertrages arbeitslos zu werden. Die Sprungbretteffekte der Leiharbeit fallen insgesamt geringer aus als die der befristeten Beschäftigung und werden durch eine gute wirtschaftliche Lage begünstigt. Bei den Minijobs stehen Brückeneffekte ganz und gar infrage, was allerdings auch damit zu tun haben dürfte, dass diese Beschäftigungsform von vornherein als Zusatzverdienst im Haushaltskontext oder als Nebenbeschäftigung angelegt ist. Die arbeitsmarktpolitische Bewertung der Brücken- und Sprungbretteffekte kann letztlich nur vor dem Hintergrund besserer Kenntnisse über weitere Arbeitsmarkteffekte atypischer Beschäftigung erfolgen. So liefern die bisherigen deskriptiven und ökonometrischen Analysen sowohl Hinweise auf Substitutionsprozesse als auch auf die Schaffung zusätzlicher Beschäftigungsmöglichkeiten. Eindeutige, kausale Belege sowie die klare Bestimmung der Größenordnung von Substitutionseffekten oder zusätzlich geschaffenen Arbeitsplätzen durch die einzelnen atypischen Beschäftigungsformen stehen jedoch noch aus und bedürfen weiterer Untersuchungen.

Deutlich wird, dass in zukünftigen empirischen Analysen simultan eine Vielzahl von möglichen Arbeitsmarkteffekten und Wechselbeziehungen zwischen den unterschiedlichen Beschäftigungsformen Berücksichtigung finden muss, um ein umfassendes Bild zu erhalten. Angesichts sinkender Arbeitslosenzahlen und hoher Beschäftigungsquoten scheint der deutsche Arbeitsmarkt aufnahmefähiger geworden zu sein. Allerdings ist seine integrative Wirkung nicht (immer) nachhaltig und hat einen Preis: Reguläre Beschäftigungsverhältnisse wurden zum Teil verdrängt, der Niedriglohnsektor ausgeweitet und die soziale Ungleichheit größer. Die Ausweitung von Minijobs, Leiharbeit und befristeter Beschäftigung hat zu einer zunehmenden Segmentierung der Beschäftigung beigetragen, wobei bestimmte Gruppen am Arbeitsmarkt, insbesondere die Geringqualifizierten, davon am stärksten betroffen sind.
Die Aufgabe der Entscheidungsträger in der Arbeitsmarktpolitik wird sein, eine nachhaltigere Beschäftigungspolitik zu entwickeln und dabei zugleich die quantitativen Erfolge am Arbeitsmarkt nicht zu gefährden. Dabei gibt es drei wesentliche Ansatzpunkte. Erstens ist zu bedenken, dass eine Eindämmung atypischer Verträge nicht unbedingt über eine arbeitsrechtliche Re-Regulierung erfolgen muss, da sie möglicherweise ins Leere läuft oder negative Auswirkungen auf den Arbeitsmarkt haben kann. Beschäftigungsverhältnisse sind eingebettet in ein Geflecht miteinander verwobener Bereiche, wie - im Falle von Minijobs - das Steuer und Sozialversicherungssystem und die staatliche Familienpolitik, oder - wie im Falle von befristeten Verträgen - die Vergabepraxis öffentlich finanzierter Dienstleistungen. Es gibt also viele alternative Stellschrauben, die Auswirkungen auf die Beschäftigungsstrukturen haben. Auch müssen Wechselwirkungen innerhalb des Beschäftigungssystems in den Blick genommen werden, da z.B. eine stärkere Regulierung einer Beschäftigungsform zu Ausweichreaktionen auf andere Beschäftigungsformen führen könnte. Zweitens geht es um Prävention, das heißt um Maßnahmen, die Bildungsarmut und Ausbildungslosigkeit soweit wie möglich vermeiden und Hochrisikogruppen in der frühen Erwerbsphase durch Beratung und Coaching bei der Arbeitsmarktintegration unterstützen. Eine dritte Option ist die konsequente Förderung von Aufwärtsmobilität, die durch eine Stärkung der Wettbewerbs- und Beschäftigungsfähigkeit erreicht werden kann. Denkbar wären etwa im Sinne eines „after care“ in der Arbeitsmarktpolitik berufsbegleitende Weiterbildungsmaßnahmen und besondere Vermittlungsbemühungen zu Gunsten von Personen, die im Niedriglohnsektor verharren oder die in spezifischer Weise von Drehtüreffekten zwischen atypischer Beschäftigung und Arbeitslosigkeit betroffen sind.

\section{LITERATUR}

Achatz, J./Bruckmeier, K./Buch, T./Burkert, C./Dietrich, H./Dietz, M./Garloff, A./ Gundert, S./Hell, S./Hohendanner, C./Jahn, E./Kleinert, C./Koller, L./Lehmer, F./Matthes, B./Niebuhr, A./ Schels, B./Trappmann, M./Walwei, U./Wapler, R./ Ziegler, K. (2012): Übergänge am Arbeitsmarkt und Qualität von Beschäftigung; in: Brücker, H. K./Möller, J./Walwei, U. (Hrsg.): Handbuch Arbeitsmarkt 2013. Analysen, Daten, Fakten, Bielefeld, S. 141-203

Bachmann, R./von den Driesch, E./Ehlert, C./Flake, R./Frings, H./Schaffner, S./ Scheuer, M. (2013): Studie zur Analyse der geringfügigen Beschäftigungsverhältnisse. Forschungsvorhaben im Auftrag des Ministeriums für Arbeit, Integration und Soziales des Landes Nordrhein-Westfalen, Essen

Baumgarten, D./Kvasnicka, M. (2012): Herausforderung Zeitarbeit. Studie im Auftrag der Bertelsmann Stiftung, Gütersloh

Bentolila, S./Saint-Paul, G. (1992): The macroeconomic impact of flexible labor contracts, with an application to Spain, in: European Economic Review 36 (5), S. $1013-1047$

Boockmann, B./Hagen, T. (2001): The Use of Flexible Working Contracts in West Germany: Evidence from an Establishment Panel, ZEW Discussion Paper 01-33, Mannheim

Boockmann, B./Hagen, T. (2005): Befristete und andere „atypische“ Beschäftigungsverhältnisse: Wird der Arbeitsmarkt funktionsfähiger?, in: Journal for Labour Market Research (Zeitschrift für ArbeitsmarktForschung) 38 (2/3) S. $305-324$

Boockmann, B./Hagen, T. (2008): Fixed-term contracts as sorting mechanisms: Evidence from job durations in West Germany, in: Labour Economics 15 (5), S. $984-1005$

Bundesagentur für Arbeit (2013): Geringfügig entlohnte Beschäftigte nach ausgewählten Merkmalen - Zeitreihe, http://statistik.arbeitsagentur.de/ Statistikdaten/Detail/Aktuel//iiia6/ sozbe-zr-geb-alter/zr-geb-alter-d-0-xls.xls (letzter Zugriff: 11.4.2013)

Caliendo, M./Wrohlich, K. (2010): Evaluating the German 'Mini-Job' reform using an natural experiment, in: Applied Economics 42 (19), S. 2475-2489 
Crimmann, A./Ziegler, K./Ellguth, P./Kohaut, S./Lehmer, F. (2009): Forschungsbericht zum Thema "Arbeitnehmerüberlassung", Nürnberg

De Cuyper, N./Jong, J./Witte, H./Isaksson, K./Rigotti, T./Schalk, R. (2008): Literature review of theory and research on the psychological impact of temporary employment: Towards a conceptual model, in: International Journal of Management Reviews 10 (1), S. 25-51

Dieckhoff, M./Steiber, N. (2012): Institutional Reforms and Age-graded Labour Market Inequalities in Europe, in: International Journal of Comparative Sociology 53 (2), S. 97-119

Dingeldey, I./Sopp, P./Wagner, A. (2012): Governance des Einkommensmix: Geringfügige Beschäftigung plus ALG-II-Bezug, in: WSI Mitteilungen 65 (1), S. 32-40, http://www.boeckler.de/wsimit_2012_01_dingeldey.pdf Freier, R./Steiner, V. (2010): 'Marginal employment' and the demand for heterogeneous labour - elasticity estimates from a multi-factor labour demand model for Germany, in: Applied Economics Letters 17 (12), S. 1177-1182 Garz, M. (2012): Employment and wages after the German 2004 reform of temporary work regulations, International Labour Review Online First Gebel, M. (2010): Early career consequences of temporary employment in Germany and the UK, in: Work, employment and society 24 (4), S. 641-660 Gebel, M. (2013): Is a temporary job better than unemployment? A cross-country comparison based on British, German, and Swiss panel data, SOEPpapers on Multidisciplinary Panel Data Research at DIW Berlin (543) Gebel, M./Giesecke, J. (2011): Labor Market Flexibility and Inequality: The Changing Skill-Biased Temporary Employment and Unemployment Risks in Europe, in: Social Forces 90 (1), S. 17-40

Giesecke, J. (2006): Arbeitsmarktflexibilisierung und soziale Ungleichheit, Wiesbaden

Giesecke, J./Groß, M. (2002): Befristete Beschäftigung: Chance oder Risiko? in: Kölner Zeitschrift für Soziologie und Sozialpsychologie 54 (1), S. 85-108 Greve, G./Pfeiffer, R./Vennebusch, T. (2007): Minijob-Zentrale - Entwicklung und Status quo der geringfügigen Beschäftigung, in: RVaktuell. Amtliches Mitteilungsblatt der Deutschen Rentenversicherung 54 (3), S. 38-43

Gundert, S. (2007): Befristete Beschäftigung bei Berufsanfängern und älteren Arbeitnehmern, Berlin

Gundert, S./Hohendanner, C. (2011): Leiharbeit und befristete Beschäftigung: Soziale Teilhabe ist eine Frage von stabilen Jobs, IAB-Kurzbericht 1/2011, Nürnberg

Hagen, T. (2003a): Do Fixed-Term Contracts Increase the Long-Term Employment Opportunities of the Unemployed?, ZEW Discussion Paper (03-49) Hagen, T. (2003b): Does Fixed-Term Contract Employment Raise Firms' Adjustment Speed? Evidence from an Establishment Panel for West Germany, in: Jahrbücher für Nationalökonomie und Statistik 223 (4), S. 403-421

Hohendanner, C. (2010): Befristete Arbeitsverträge zwischen Auf- und Abschwung: Unsichere Zeiten, unsichere Verträge?, IAB-Kurzbericht 14/2010, Nürnberg

Hohendanner, C. (2012): Churning im Kontext betrieblicher Personalpolitik. Ak tuelle Entwicklungen der Beschäftigungssysteme, in: Industrielle Beziehungen 19 (2), S. $124-153$

Hohendanner, C./Gerner, H.-D. (2010): Die Übernahme befristet Beschäftigter im Kontext betrieblicher Personalpolitik, in: Soziale Welt 61 (1), S. 27-50 Hohendanner, C./Stegmaier, J. (2012): Umstrittene Minijobs. Geringfügige Beschäftigung in deutschen Betrieben, IAB-Kurzbericht 24/2012, Nürnberg Jacobi, L./Schaffner, S. (2008): Does Marginal Employment Substitute Regular Employment? - A Heterogeneous Dynamic Labor Demand Approach for Germany, Ruhr Economic Papers (56)

Jahn, E./Bentzen, J. (2012): What drives the demand for temporary agency workers?, in: Labour 26 (3), S. 341-355

Jahn, E./Weber, E. (2013): Zeitarbeit. Zusätzliche Jobs, aber auch Verdrängung, IAB-Kurzbericht 2/2013, Nürnberg

Kahn, L. M. (2010): Employment protection reforms, employment and the incidence of temporary jobs in Europe: 1996-2001, in: Labour Economics 17 (1), S. $1-15$

Kalina, T./Voss-Dahm, D. (2005): Mehr Minijobs = mehr Bewegung auf dem Arbeitsmarkt? Fluktuation der Arbeitskräfte und Beschäftigungsstruktur in vier Dienstleistungsbranchen, IAT-Report 2005-07, Gelsenkirchen
Keller, B./Seifert, H./Schulz, S./Zimmer, B. (2011): Atypische Beschäftigung und soziale Risiken. Entwicklung, Strukturen, Regulierung, WISO Diskurs, Expertise im Auftrag der Friedrich-Ebert-Stiftung, Bonn

Körner, T./Meinken, H./Puch, K. (2013): Wer sind die ausschließlich geringfügig Beschäftigten? Eine Analyse nach sozialer Lebenslage, in: Wirtschaft und Statistik (1) S. 42-61

Kvasnicka, M. (2008): Does Temporary Help Work Provide a Stepping Stone to Regular Employment?, NBER Working Paper (13843)

Lehmer, F. (2012): Dient die Arbeitnehmerüberlassung für Langzeitarbeitslose als Brücke in nachhaltige Beschäftigung?, in: Sozialer Fortschritt 61 (8), S. $190-197$

Lehmer, F./Ziegler, K. (2010): Brückenfunktion der Leiharbeit. Zumindest ein schmaler Steg, IAB-Kurzbericht 13/2010, Nürnberg

McGinnity, F./Mertens, A./Gundert, S. (2005): A Bad Start? Fixed-Term Contracts and the Transition from Education to Work in West Germany, in: European Sociological Review 21 (4), S. 359-374

Möller, J./Walwei, U./Ziegler, K. (2012): Wie wichtig ist Zeitarbeit für den deutschen Arbeitsmarkt?, in: Dinges, A./Franken, H./Breucker, G./Calasan, V./ Speidel, C. (Hrsg.): Zukunft Zeitarbeit. Perspektiven für Wirtschaft und Gesellschaft, Berlin, S. 33-53

Nunziata, L./Staffolani, S. (2007): Short-term contracts regulation and dynamic labour demand. Theory and evidence, in: Scottish Journal of Political Economy 54 (1), S. $72-104$

Pfeifer, C. (2009): Fixed-term Contracts and Employment Adjustment: An Empirical Test of the Core-Periphery Hypothesis Using German Establishment Data, in:The Economic Record 85 (268), S. 92-107

Promberger, M. (2012): Topographie der Leiharbeit. Flexibilität und Prekarität einer atypischen Beschäftigungsform, Berlin

Rudolph, H. (2006): Neue Beschäftigungsformen: Brücken aus der Arbeitslosigkeit?, in: Badura, B./Schellschmidt, H./Vetter, C. (Hrsg.): Fehlzeiten-Report 2005. Arbeitsplatzunsicherheit und Gesundheit. Zahlen, Daten, Analysen aus allen Branchen der Wirtschaft, Berlin, S. 35-56

Sachverständigenrat (2009): Jahresgutachten 2008/2009: Die Finanzkrise meistern - Wachstumkräfte stärken, Wiesbaden

Schmidt, K./Wüllerich, J. (2013): Zeitarbeit in Deutschland - Aktuelle Entwicklungen. Der Arbeitsmarkt in Deutschland. Arbeitsmarktberichterstattung der Bundesagentur für Arbeit, Nürnberg

Voss, D./Weinkopf, C. (2012): Niedriglohnfalle Minijob, in: WSI-Mitteilungen 65 (1), S. 5-12, http://www.boeckler.de/wsimit_2012_01_voss.pdf

Wippermann, C. (2012): Frauen im Minijob - Motive und (Fehl-)Anreize für die Aufnahme geringfügiger Beschäftigung im Lebenslauf: Bundesministerium für Familie, Frauen, Senioren und Jugend, Berlin

\section{AUTOREN}

CHRISTIAN HOHENDANNER, Dr., ist Wissenschaftlicher Mitarbeiter am Institut für Arbeitsmarkt- und Berufsforschung (IAB) Nürnberg. Arbeitsschwerpunkte: Wandel der Beschäftigungsstrukturen, Qualität der Beschäftigung, betriebliche Personalpolitik, aktive Arbeitsmarktpolitik.

christian.hohendanner@iab.de

ULRICH WALWEI, Dr., ist Vizedirektor des Instituts für Arbeitsmarkt- und Berufsforschung (IAB) Nürnberg. Arbeitsschwerpunkte: längerfristige Arbeitsmarkttrends, Institutionenökonomik, Politikanalysen, Flexibilisierung des Arbeitsmarktes.

ulrich.walwei@iab.de 